\title{
Mediastinal myxoid neurofibroma: unusual location of a rare disease
}

\begin{abstract}
Myxoid neurofibroma (MN) is a benign tumor of the peripheral nerve arising from connective tissue cells. To our knowledge mediastinal location of $\mathrm{MN}$ has not yet been previously reported in the medical literature. Through observation we discuss the management of this rare entity. A 48 year old female with no known medical history was admitted in our medical center and underwent a complete resection of a compressive mediastinal tumor mass. Pathological examination carried out by two different laboratories confirmed that it was a myxoid neurofibroma of the mediastinum. Although rare, mediastinal location of myxoid neurofibroma should be considered and included in the differential diagnosis of mediastinal tumors. Surgical management is based on total resection and is the only guarantee of a good prognosis.
\end{abstract}

Keywords: neurofibroma, mediastinum, myxoid tumor, thoracotomy
Volume 4 Issue 3 - 2017

\author{
Issoufou I,' Ouadnouni Y, ${ }^{1,2}$ Smahi $M^{1,2}$ \\ 'Thoracic Surgery, Teaching Hospital Hassan II, Morocco \\ ${ }^{2}$ University Sidi Mohamed Ben Adellah, Faculty of Medicine and \\ Pharmacy, Morocco
}

Correspondence: Issoufou Ibrahim, Thoracic Surgery, Teaching Hospital Hassan II, BP 1893, Km 2.200, Siditharazem Road, Fez 30000, Morocco, Tel +212-6-97-12-55-26,

Email alzoumib84@gmail.com

Received: August 05, 2017 | Published: August 22, 2017

\section{Introduction}

Myxoid neurofibroma (MN) is a benign tumor of the peripheral nerves arising from connective tissue cells. This is a rare tumor characterized by immunostaining for $\mathrm{S}-100$ protein. ${ }^{1}$ From its nervous origin, its topography is fairly diverse. The most common locations of the $\mathrm{MN}$ are the face, shoulders, arms, periungual and in the feet. Mediastinal location has not yet been described. Through observation, to our knowledge, we report the first case of mediastinal location of a myxoid neurofibroma and discuss the diagnostic approach.

\section{Case report}

A woman aged 48 , with no notable medical history was referred to our medical service for atypical chest pain and experiencing dyspnea, Sadoul stage II, for 3 months. The clinical examination revealed a patient in good condition with a left pulmonary consolidation on chest x-ray (CXR). The dermatological examination was unremarkable. Chest radiography showed an opacity occupying the lower half of the left hemi thorax extending to right para-cardiac area (Figure 1). Chest CT (Figures 2A \& 2B) showed an anterior mediastinalmass with a heterogeneous tissue density image with an exothoracic component, without signs of parietal invasion. This mass was compressing the heart chambers and supra-cardiac vessels without invasion. Laboratory tests included the assay of alpha-fetoprotein and beta HCG and were both negative. An earlier mediastinotomy exploration was performed. The exploration revealed an encapsulated tumor/tissue component with gelatinous appearance that suggested a huge bronchogenic cyst. The indication for surgical resection had been opted without carrying out a prior biopsy. Thoracotomy through the anterolateral left $4^{\text {th }}$ intercostal space was performed. The exploration revealed an encapsulated anterior mediastinal tumor with an exothoracic component creeping into the $2^{\text {nd }}$ left intercostal space without parietal or mediastinal invasion. Complete tumor resection including the exothoracic component was performed. Pathological examination (Figures 3-5) of the tumor tissue undertaken by two different laboratories confirmed the diagnosis of benign myxoid mediastinal neurofibroma. The postoperative course was uneventful, the patient was discharged post-operatively on day 5 .

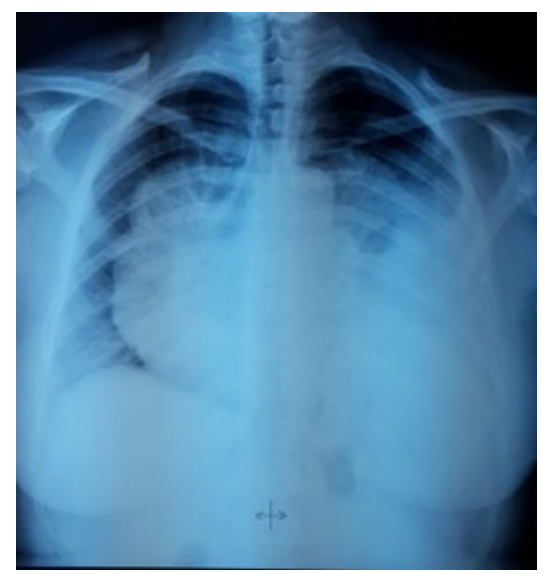

Figure I Opacity occupying the lower half of the left hemithorax extending to right para-cardiac area.

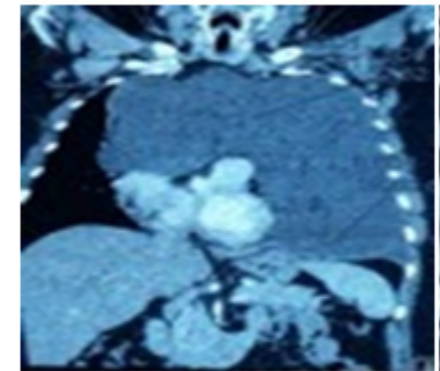

$2 \mathrm{~A}$

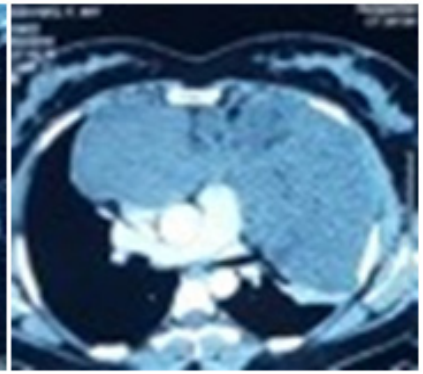

$2 \mathrm{~B}$
Figures 2A \& 2B Anterior mediastinal mass with heterogeneous tissue density image with exothoracic component without signs of parietal invasion. This mass is compressing the heart chambers and repressed supra-cardiac vessels without invasion. 


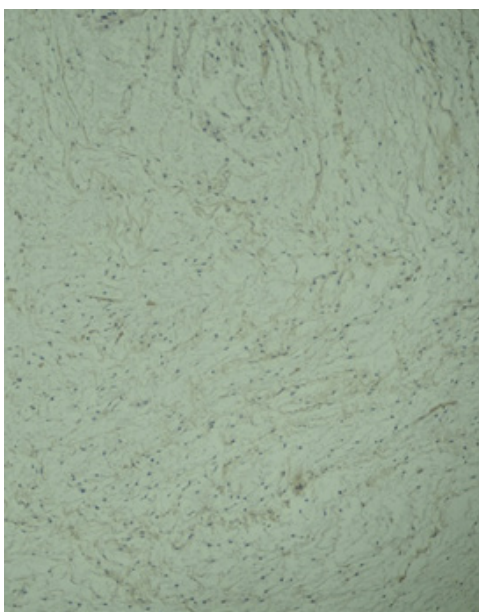

Figure 3 Myxoid neurofibroma: myxoidtumor of fusocellular architecture with collagenous and few vascularisation $(x \mid 0)$.

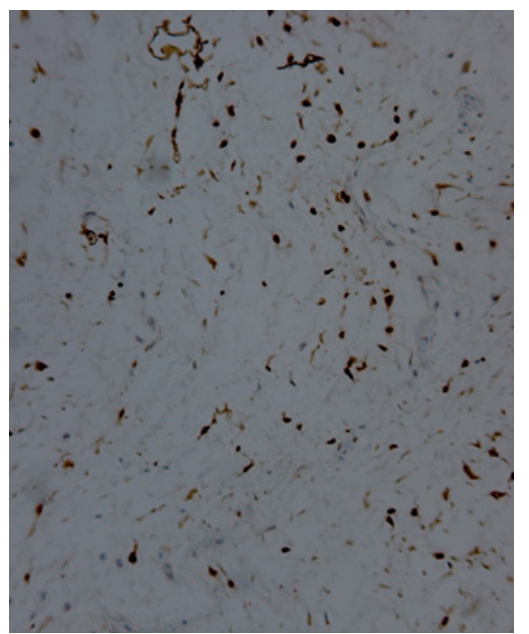

Figure 4 Myxoid neurofibroma: absence of cytotoxic atypia or mitosis (x20).

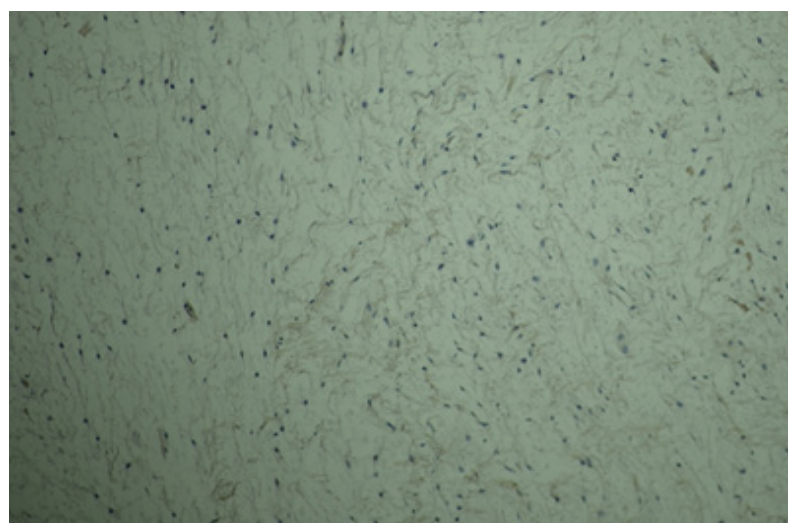

Figure 5 Myxoid neurofibroma: diffuse and intense nuclear marking by the PS $100(\times 20)$

\section{Discussion}

Myxoid neurofibroma is a rare tumor. It usually occurs on the face, shoulders, arms, around the nails and feet. ${ }^{2}$ Unlike these locations most of which are asymptomatic, ${ }^{2,3}$ the mediastinal location is of particular importance because of its proximity to the mediastinal organs.
The symptomatology is dominated by chest pain, cough, dyspnea, dysphagia or superior vena cava syndrome. The CT scan features are that of a mediastinal mass compressing or displacing structures without invasion. In our patient it revealed a large heterogeneous mass of tissue with cystic components occupying the anterior mediastinum with enhancement after contrast injection and compressing the mediastinum and both lungs without signs of invasion. To our knowledge this is the first case of mediastinal location of a myxoid neurofibroma. It poses a problem of differential diagnosis with anterior mediastinal masses including thymic epithelial tumors and lymphomas. Thus, certainty of the diagnosis is based on histology. This diagnosis is a challenge to the pathologist for two reasons. Firstly, because of many diagnostic possibilities (egg. an aggressive angiomyxoma, fibromyxoidsarcoma, low-grade myxoidliposarcoma etc.). ${ }^{4}$ Secondly, biopsy fragments that are small do not allow a full pathological and immunohistochemical study. ${ }^{5}$ Hence it is now important to include myxoid neurofibroma in the differential diagnosis and to make earlier mediastinotomy biopsies for confirmation of the proper diagnosis. In the case of our patient, the first exploration before completion of the biopsy revealed a resectable encapsulated mobile anterior mediastinal tumor, resulting in a complete tumor resection without any biopsy. Indeed, this resection remains the only therapeutic approach in myxoid neurofibroma. One must take out the tumor in its entirety to prevent recurrence. ${ }^{1}$ This must be performed in view of clinical signs of mediastinal compression and should be considered as early as possible to avoid the occurrence of superior vena cava syndrome with thromboembolic complications. The choice of surgical approach will depend primarily on the location of the tumor. In our patient, it was a left anterolateral thoracotomy. It allowed complete resection of the tumor despite its extension in the right hemi-thorax. A clamshell approach could also be carried out but it was deemed unsuitable in this case. Although myxoid neurofibroma is a rare disease and unusual in the chest, mediastinal location must be thought of and now considered and included in the differential diagnosis of mediastinal masses. Its management is surgical and based on total resection as the only guarantee of a good prognosis.

\section{Acknowledgments}

None.

\section{Conflicts of interest}

The authors declare that there is no conflict of interest regarding the publication of this paper.

\section{Funding}

None.

\section{References}

1. Hocar O, Madhar M, Akhdari N, et al. Neurofibrome myxoïde du pouce. Chir Main. 2012;31(4):217-219.

2. Ponce-Olivera RM, Tirado-Sanchez A, Peniche-Castellanos A, et al. Myxoid neurofibroma: an unusual presentation. Indian J Dermatol. 2008;53(1):35-36.

3. Gmyrek RF, Beer R, Silvers DN, et al. Periungual myxoid neurofibroma. Cutis. 2002;69(1):54-56.

4. Chennakeshaviah G, Ravishankar S, Maggad R, et al. Solitary giant intramuscular myxoid neurofibroma resulting in an above elbow amputation. Case Rep Pathol. 2012.

5. Labonté S. Les tumeurs myxoïdes des tissus mous profonds. Ann Pathol. 2015;35(1):3-14. 